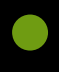 \\ hree \\ a journal of mathematics
}

A topological generalization of partition regularity

Liam Solus

mathematical sciences publishers 


\title{
A topological generalization of partition regularity
}

\section{Liam Solus}

\author{
(Communicated by Chi-Kwong Li)
}

\begin{abstract}
In 1939, Richard Rado showed that any complex matrix is partition regular over $\mathbb{C}$ if and only if it satisfies the columns condition. Recently, Hogben and McLeod explored the linear algebraic properties of matrices satisfying partition regularity. We further the discourse by generalizing the notion of partition regularity beyond systems of linear equations to topological surfaces and graphs. We begin by defining, for an arbitrary matrix $\Phi$, the metric space $\left(M_{\Phi}, \delta\right)$. Here, $M_{\Phi}$ is the set of all matrices equivalent to $\Phi$ that are (not) partition regular if $\Phi$ is (not) partition regular; and for elementary matrices, $E_{i}$ and $F_{j}$, we let $\delta(A, B)=$ $\min \left\{m=l+k: B=E_{1} \ldots E_{l} A F_{1} \ldots F_{k}\right\}$. Subsequently, we illustrate that partition regularity is in fact a local property in the topological sense, and uncover some of the properties of partition regularity from this perspective. We then use these properties to establish that all compact topological surfaces are partition regular.
\end{abstract}

\section{Introduction}

Let $\mathbb{C}$ be the set of complex numbers, and let $\mathbb{M}_{u, v}(\mathbb{C})$ be the set of all $u \times v$ matrices with complex entries. Let $A=\left[a_{i, j}\right] \in \mathbb{M}_{u, v}(\mathbb{C})$ be given, and let $\vec{a}_{j}$ denote the column $j$ of $A$. Then $A$ satisfies the columns condition if and only if there exists an $m \in\{1, \ldots, v\}$ and a partition $\left\{I_{1}, \ldots, I_{m}\right\}$ of $\{1, \ldots, v\}$ into nonempty sets such that

(i) $\sum_{j \in I_{1}} \vec{a}_{j}=\overline{\mathbf{0}}$, and

(ii) for each $t \in\{2,3, \ldots, m\}$ (if any), $\sum_{i \in I_{t}} \vec{a}_{i}$ is in the span of $\left\{\vec{a}_{i}: i \in \bigcup_{j=1}^{t-1} I_{j}\right\}$.

$A$ is said to be partition regular if it satisfies the columns condition [Hindman 2007; Rado 1943]. The study of partition regularity has long been a combinatorial endeavor, which mostly uses the columns condition to check if a given matrix is partition regular. However, Hogben and McLeod [2010] recently showed that the columns condition is interesting in its own right, and provided a more linear

MSC2000: primary 05C99, 05E99, 15A06, 54H10, 57N05; secondary 15A99, 54E35.

Keywords: partition regularity, columns condition, graphs, metric space, discrete topology, topological surface, triangulation. 
algebraic perspective on partition regularity. We employ this new perspective to extend the notion of partition regularity into geometrical and topological settings.

For an arbitrary complex matrix $\Phi$, we construct a metric space characterized by the partition regularity of $\Phi$ (Section 2). We then use this metric space about $\Phi$ to generate a topological space that recasts partition regularity as a local property. We show a few topological properties of these spaces, and then demonstrate how their systems of neighborhoods can describe the "degree" of partition regularity as applied to a given matrix (Section 2). Finally, using some well known connections between graph theory and linear algebra, we construct topological spaces that allow us to define partition regularity as a property of topological surfaces and graphs. In Section 3, we show that all compact topological surfaces are partition regular. We then demonstrate that not all graphs are partition regular.

We take a topological surface to be a two-dimensional real manifold that is Hausdorff. A graph $G=(V, E)$ is a nonempty set $V$ of vertices, along with a set $E$ of edges, where an edge is a two-element subset of vertices. A walk is an alternating sequence $\left(v_{0}, e_{1}, v_{1}, e_{2}, \ldots, e_{m}, v_{m}\right)$ of vertices and edges. A graph $G$ is connected if there exists a walk between any two distinct vertices of $G$. A component is a connected subgraph of $G$, and a set $S$ of edges of $G$ is a disconnecting set if $G \backslash S$ has more than one component. The edge connectivity of $G$ is the minimum size of a disconnecting set of $G$. An orientation $\Gamma$ of $G$ is obtained by assigning a direction to each edge of $G$, and thus replacing the edge $\{i, j\}$ with the $\operatorname{arc}(i, j)$. An orientation $\Gamma$ of $G$ is strongly connected if there exists an alternating sequence $\left(v_{0}, e_{1}, v_{1}, e_{2}, \ldots, e_{m}, v_{m}\right)$ of vertices and arcs between any two vertices of $\Gamma$. The oriented incidence matrix of $\Gamma$ is the rational matrix denoted $D_{\Gamma}=\left[d_{i, e}\right]$, where if $e=(i, j)$, then $d_{i, e}=-1, d_{j, e}=1$, and $d_{k, e}=0$ for $k \neq i$ and $k \neq j$.

For any matrix $A$ in $\mathbb{M}_{m, n}(\mathbb{C}$ ), we let a type-1 elementary operation be a row (column) permutation, a type-2 elementary operation be multiplication of a given row (column) of $A$ by a scalar $\beta \in \mathbb{C}$, and a type-3 elementary operation be the addition of a scalar multiple of one row (column) of $A$ to another. We call the associated matrices of each elementary operation $\mathrm{T} 1, \mathrm{~T} 2$, and $\mathrm{T} 3$ matrices, respectively.

\section{Topologically rich spaces associated with partition regularity}

Let $A, B \in \mathbb{M}_{m, n}(\mathbb{C})$. We say that $B$ is equivalent to $A$ if there exist invertible matrices $P$ and $Q$ for which $B=P A Q$. This is an equivalence relation on $\mathbb{M}_{m, n}(\mathbb{C})$, and we let $[A]$ denote the equivalence class of $A$. Since $P$ and $Q$ are each the product of a finite number of elementary matrices we can identify $P$ and $Q$, with the sequence of nonidentity elementary matrices $\langle x\rangle_{i=1}^{l}$ that when applied to matrix $A$ produces matrix $B$. Since $A$ and $B$ are both in $[A]$, there must exist a minimal sequence of elementary operations. Let $l_{A, B}$ be the nonnegative integer denoting 
the length of this minimal sequence. Then we can define the function

$$
\delta:[A] \times[A] \longrightarrow \mathbb{R}
$$

such that

$$
\delta(A, B)=l_{A, B} .
$$

Theorem 2.1. Let $A$ be in $\mathbb{M}_{m, n}(\mathbb{C})$. Then $([A], \delta)$ is a metric space.

Proof. The nonnegativity of $\delta$ follows trivially from the definition of $l_{A, B}$ for any pair of matrices $A, B$ in $[A]$.

To see that $\delta$ is symmetric, notice that if $\delta(A, B)=l_{A, B}$, then there exist invertible matrices $P$ and $Q$ associated with the minimal sequence $\langle x\rangle_{i=1}^{l_{A, B}}$ such that $B=P A Q$. It follows that $A=P^{-1} B Q^{-1}$, and thus $P^{-1}$ and $Q^{-1}$ may be associated with the sequence $\langle x\rangle_{i=1}^{m}$, where $m=l_{A, B}$ is equal to the number of elementary matrices in the product $P^{-1} Q^{-1}$. Assume that $\langle x\rangle_{i=1}^{m}$ is not minimal, then there must exist a sequence $\langle x\rangle_{i=1}^{l_{B, A}}$ such that $l_{B, A}<m$. Find its associated invertible matrices $U$ and $V$ such that $A=U B V$. So, $B=U^{-1} A V^{-1}$ and there is an associated sequence $\langle x\rangle_{i=1}^{n}$. But $n=l_{B, A}$, and since $m=l_{A, B}$, this contradicts the minimality of $\langle x\rangle_{i=1}^{l_{A, B}}$. Thus,

$$
\delta(A, B)=l_{A, B}=m=l_{B, A}=\delta(B, A),
$$

and $\delta$ is a symmetric function.

To see that $\delta$ satisfies the triangle inequality, let $A, B$, and $C$ be in $[A]$, and pick $G, P, U, V, N$, and $Q$ such that $B=G A P, C=U A V$, and $B=N C Q$. Then

$$
\delta(A, B)=l_{A, B}, \quad \delta(A, C)=l_{A, C}, \quad \delta(C, B)=l_{C, B} .
$$

Now let $m=\delta(A, C)+\delta(C, B)$. Then there exists a sequence $\langle x\rangle_{i=1}^{m}$ associated with the invertible matrices $N U$ and $V Q$ such that

$$
B=(N U) A(V Q) .
$$

Thus, since $A$ can be changed to $B$ using $\delta(A, C)+\delta(C, B)$ elementary operations, it follows from the minimality of $\delta(A, B)$ that

$$
\delta(A, B) \leq \delta(A, C)+\delta(C, B) .
$$

So, $\delta$ satisfies the triangle inequality, and $([A], \delta)$ is indeed a metric space.

Let $\Phi \in \mathbb{M}_{m, n}(\mathbb{C})$ be a partition regular matrix, and let $M_{\Phi}$ denote the set of all matrices that are partition regular and equivalent to $\Phi$. Notice that $\left(M_{\Phi}, \delta\right)$ is a metric space. Furthermore, the range of our metric $\delta$ is a subset of the nonnegative integers.

Theorem 2.2. Let $\mathscr{T}$ be the metric topology induced by $\delta$ on $M_{\Phi}$. Then $\left(M_{\Phi}, \mathscr{T}\right)$ is a discrete topological space. 
Proof. Let $A, B \in \mathbb{M}_{m, n}(\mathbb{C})$. We need only prove that $\delta(A, B)=0$ if and only if $A=B$. To see this, notice that for any $A, B$ in $M_{\Phi}, \delta(A, B)=l_{A, B}$ is a nonnegative integer. So, if $\delta(A, B)=0$, then the minimal number of nonidentity elementary matrices that must be applied to $A$ to produce $B$ is zero. So it must be that $A=B$. Conversely, if $A=B$, then the minimal number of elementary operations that must be applied to $A$ to reach $B$ is equal to 0 . Thus, $\delta(A, B)=0$.

For $A_{0}$ in $M_{\Phi}$ consider the open ball of radius $\frac{1}{2}$ about $A_{0}$ :

$$
\mathscr{P}_{A_{0}, 1 / 2}=\left\{A \in M_{\Phi}: \delta\left(A_{0}, A\right)<\frac{1}{2}\right\}=\left\{A \in M_{\Phi}: \delta\left(A_{0}, A\right)=0\right\}=\left\{A_{0}\right\} .
$$

Therefore, the singletons of $M_{\Phi}$ are all open sets, and so $\mathscr{T}$ is equal to the power set of $M_{\Phi}$. Thus, the pair $\left(M_{\Phi}, \mathscr{T}\right)$ is a discrete topological space.

Notice that if $\Phi$ is not partition regular, then there is a corresponding discrete space consisting of all matrices that are not partition regular and equivalent to $\Phi$. This allows us to establish a "degree" of partition regularity for any arbitrary matrix.

Definition 2.3. Let $A \in \mathbb{M}_{u, v}(\mathbb{C})$ be given.

(a) The progress of $A$ is the minimum number, $l$, of elementary operations that must be performed on $A$ to produce a partition regular matrix. We say that $A$ has progress $l$, and write $\operatorname{pr}(A)=l$, moreover, we write $\operatorname{pr}(A)=\infty$ if $A$ cannot be changed into a partition regular matrix via elementary operations.

(b) The antiprogress of $A$ is the minimum number, $l$, of elementary operations that must be performed on $A$ to produce a matrix that is not partition regular. We say that $A$ has antiprogress $l$, and write $\operatorname{apr}(A)=l$, moreover, we write $\operatorname{apr}(A)=\infty$ if $A$ cannot be changed into a matrix that is not partition regular via elementary operations.

Any $A \in \mathbb{M}_{u, v}(\mathbb{C})$ has both a progress and an antiprogress. Moreover, $A$ has progress 0 if and only if $A$ is partition regular, and $A$ has antiprogress 0 if and only if $A$ is not partition regular.

We are interested in the collection of matrices that proceed from a given matrix $A$ in $M_{\Phi}$. The following definitions describe such collections.

Definition 2.4. (a) A filament is a sequence of equivalent matrices in $M_{\Phi}$ satisfying the following conditions:

(i) The sequence begins with $\Phi$.

(ii) No matrix in the sequence is repeated.

(iii) The sequence is finite if and only if the last matrix in the sequence has antiprogress 1.

(iv) Each matrix of the sequence is obtained by performing a single elementary operation on the preceding matrix in the sequence. 
A filament is called finite if the sequence is finite. Otherwise, it is called infinite.

(b) A subfilament associated with $A$ is a sequence of equivalent matrices in $M_{\Phi}$ starting with matrix $A$, that satisfies (ii), (iii), and (iv). A subfilament is called finite if the sequence is finite. Otherwise, it is called infinite.

Example 2.5. Let $\Phi=\left[\begin{array}{rrr}-1 & 1 & 0 \\ -1 & 0 & 1 \\ 0 & 0 & 0\end{array}\right]$. Then

$$
\left(\left[\begin{array}{rrr}
-1 & 1 & 0 \\
-1 & 0 & 1 \\
0 & 0 & 0
\end{array}\right],\left[\begin{array}{lll}
-1 & 1 & 0 \\
-1 & 0 & 1 \\
-1 & 1 & 0
\end{array}\right]\right) \text { and }\left(\left[\begin{array}{rrr}
-1 & 1 & 0 \\
-1 & 0 & 1 \\
0 & 0 & 0
\end{array}\right],\left[\begin{array}{lll}
-1 & 1 & 0 \\
-1 & 0 & 1 \\
-1 & 1 & 0
\end{array}\right],\left[\begin{array}{rrr}
-1 & 1 & 0 \\
-1 & 0 & 1 \\
0 & 1 & -1
\end{array}\right]\right)
$$

are finite filaments in $M_{\Phi}$ since

$$
\left[\begin{array}{lll}
-1 & 1 & 0 \\
-1 & 0 & 1 \\
-1 & 1 & 0
\end{array}\right]\left[\begin{array}{rrr}
-1 & 0 & 0 \\
0 & 1 & 0 \\
0 & 0 & 1
\end{array}\right]=\left[\begin{array}{lll}
1 & 1 & 0 \\
1 & 0 & 1 \\
1 & 1 & 0
\end{array}\right] \text { and }\left[\begin{array}{rrr}
-1 & 1 & 0 \\
-1 & 0 & 1 \\
0 & 1 & -1
\end{array}\right]\left[\begin{array}{rrr}
-1 & 0 & 0 \\
0 & 1 & 0 \\
0 & 0 & 1
\end{array}\right]=\left[\begin{array}{lll}
1 & 1 & 0 \\
1 & 0 & 1 \\
0 & 1 & 0
\end{array}\right]
$$

are both not partition regular. If $A=\left[\begin{array}{lll}-1 & 1 & 0 \\ -1 & 0 & 1 \\ -1 & 1 & 0\end{array}\right]$, then

$$
\left(\left[\begin{array}{rrr}
-1 & 1 & 0 \\
-1 & 0 & 1 \\
-1 & 1 & 0
\end{array}\right],\left[\begin{array}{rrr}
-1 & 1 & 0 \\
-1 & 0 & 1 \\
0 & 1 & -1
\end{array}\right]\right)
$$

is a finite subfilament associated with $A$.

The following theorem provides a better description of exactly how the size of $M_{\Phi}$ relates to the degree of partition regularity of $\Phi$.

Theorem 2.6. For any partition regular matrix $\Phi$ in $\mathbb{M}_{m, n}(\mathbb{C}), M_{\Phi}$ contains an infinite number of infinite filaments.

Proof. Since row equivalent matrices share the same nullspace, we have, as an immediate consequence of [Hogben and McLeod 2010, Theorem 2.3], that partition regularity is invariant under elementary row operations. It follows from the definition of the columns condition that partition regularity is also invariant under type-1 column operations. Thus, there exist exactly four types of elementary operations that cannot produce a matrix that has antiprogress 0 from a partition regular matrix. For any $A \in M_{\Phi}$ we may apply a type-1 row operation for every possible pair of rows in $A$, and similarly for any type-1 column operation. This gives $\left(\begin{array}{c}m \\ 2\end{array}\right)$ possible type 1 row operations, and $\left(\begin{array}{l}n \\ 2\end{array}\right)$ possible type- 1 column operations that can be applied to $A$ and produce a matrix in $M_{\Phi}$. type- 2 row operations also cannot produce a matrix that has antiprogress 0 from $A$. Since any scalar in $\mathbb{C}-\{0\}$ may be applied to a single row of $A$, there exist $m|\mathbb{C}-\{0\}|$ possible type-2 row operations that can be applied to $A$. Finally, there are $|\mathbb{C}-\{0\}|$ ways to scale a given row of $A$, and $m-1$ rows to which this scaled row may be added. Since this may be done 
for any row of $A$, there exist $m(m-1)|\mathbb{C}-\{0\}|$ type-3 row operations that can be applied to $A$ and can produce a matrix in $M_{\Phi}$. Thus, for any $A \in M_{\Phi}$, there are exactly

$$
m(m-1)|\mathbb{C}-\{0\}|+m|\mathbb{C}-\{0\}|+\left(\begin{array}{c}
m \\
2
\end{array}\right)+\left(\begin{array}{l}
n \\
2
\end{array}\right)
$$

elementary operations that will not produce a matrix that has antiprogress 0 when applied to $A$. Thus, for each matrix produced by applying exactly these operations to $\Phi$, we can produce another $m(m-1)|\mathbb{C}-\{0\}|+m|\mathbb{C}-\{0\}|+\left(\begin{array}{c}m \\ 2\end{array}\right)+\left(\begin{array}{l}n \\ 2\end{array}\right)$ that are still partition regular. Continuing in this fashion, we produce

$$
\left[m(m-1)|\mathbb{C}-\{0\}|+m|\mathbb{C}-\{0\}|+\left(\begin{array}{c}
m \\
2
\end{array}\right)+\left(\begin{array}{l}
n \\
2
\end{array}\right)\right]^{\aleph_{0}}
$$

infinite sequences of matrices contained in $M_{\Phi}$.

Now, if

$$
\left(\Phi, A_{1}, A_{2}, A_{3}, \ldots\right)
$$

is an infinite sequence of matrices in $M_{\Phi}$, where each $A_{i}$ is produced by applying exactly one of the infinitely many operations described above to $A_{i-1}$, then there exist only a finite subset of these operations such that $A_{i}=A_{j}$ for some

$$
A_{j} \in\left\{\Phi, A_{1}, \ldots, A_{i-1}\right\} .
$$

Notice that only the identity operation may be applied to $A_{i-1}$ to produce $A_{i-1}$. Then to see the result, assume there exists some elementary operation $E_{0}$ such that $E_{0}$ applied to $A_{i-1}$ produces $A_{i} \in M_{\Phi}$ and

$$
A_{i} \in\left\{\Phi, A_{1}, \ldots, A_{i-2}\right\} .
$$

If $E_{0}$ is a type- 1 row operation, there exists some

$$
A_{j} \in\left\{\Phi, A_{1}, \ldots, A_{i-2}\right\}
$$

such that switching exactly two rows of $A_{j}$ produces $A_{i-1}$. Since there exist for each

$$
A_{j} \in\left\{\Phi, A_{1}, \ldots, A_{i-2}\right\}
$$

exactly two rows that may be switched to produce $A_{i-1}$, there exist at most $(i-1)$ possibilities for $E_{0}$ to be a type-1 row operation such that

$$
A_{i}=E_{0} A_{i-1} \in\left\{\Phi, A_{1}, \ldots, A_{i-2}\right\} .
$$

Similarly, there exist at most $(i-1)$ possibilities for $E_{0}$ to be a type-1 column operation such that

$$
A_{i}=A_{i-1} E_{0} \in\left\{\Phi, A_{1}, \ldots, A_{i-2}\right\} .
$$


In the case that $E_{0}$ is a type- 2 row operation, and

$$
E_{0} A_{i-1} \in\left\{\Phi, A_{1}, \ldots, A_{i-2}\right\}
$$

then we may produce some $A_{j} \in\left\{\Phi, A_{1}, \ldots, A_{i-2}\right\}$ by scaling exactly one row of $A_{i-1}$ by a single $\alpha \in \mathbb{C}$. Since $\mathbb{C}$ is a unique factorization domain, it follows that if $E_{0} A_{i-1}=A_{j}, E_{0}$ is unique. Therefore, at most, we may find one $E_{0}$ such that $E_{0} A_{i-1}=A_{j}$ for each $A_{j} \in\left\{\Phi, A_{1}, \ldots, A_{i-2}\right\}$. Thus, there are at most $(i-1)$ type-2 column operations such that $A_{i} \in\left\{\Phi, A_{1}, \ldots, A_{i-2}\right\}$.

Finally, let $E_{0}$ be a type-3 column operation such that

$$
E_{0} A_{i-1}=A_{j} \in\left\{\Phi, A_{1}, \ldots, A_{i-2}\right\} .
$$

Then exactly one row of $A_{j}$, call it row $\left(A_{j}\right)_{k}$, is not equal to row $\left(A_{i-1}\right)_{k}$, and

$$
\left(A_{j}\right)_{k}=\alpha\left(A_{i-1}\right)_{k^{\prime}}+\left(A_{i-1}\right)_{k}
$$

where $\alpha \in \mathbb{C}$, and $\left(A_{i-1}\right)_{k^{\prime}}$ is one of the $(m-1)$ rows of $A_{i-1}$ that is not row $\left(A_{i-1}\right)_{k}$. It follows that

$$
\left(A_{j}\right)_{k}-\left(A_{i-1}\right)_{k}=\alpha\left(A_{i-1}\right)_{k^{\prime}}
$$

and again $\alpha \in \mathbb{C}$ must be unique. Thus, for a given

$$
A_{j} \in\left\{\Phi, A_{1}, \ldots, A_{i-2}\right\}
$$

there are at most $(m-1)$ possibilities for $E_{0}$ such that $E_{0} A_{i-1}=A_{j}$. Therefore, there are at most $(m-1)(i-1)$ possibilities for $E_{0}$ to be a type- 3 column operation such that $A_{i} \in\left\{\Phi, A_{1}, \ldots, A_{i-2}\right\}$.

We may now conclude from these various cases that there are at most

$$
3(i-1)+(m-1)(i-1)+1
$$

elementary operations that when applied to $A_{i-1}$ will yield for $A_{i}$ an element of $\left\{\Phi, A_{1}, \ldots, A_{i-1}\right\}$. Thus, for each $A_{i}$ in the sequence there still exist an infinite number of elementary operations such that $A_{i+1} \in M_{\Phi}$ and $A_{i+1} \notin\left\{\Phi, A_{1}, \ldots, A_{i}\right\}$. It follows that there exist an infinite number of infinite filaments in $M_{\Phi}$, for any partition regular matrix $\Phi$.

For any partition regular matrix, $\Phi$, the space $M_{\Phi}$ is large. Moreover, for any two partition regular matrices, $\Phi$ and $\Psi$, the cardinality of the collection of infinite filaments in $M_{\Phi}$ is the same as the cardinality of the collection of infinite filaments in $M_{\Psi}$. This makes it difficult to use the size of these spaces to say that one matrix is "more" partition regular than another. 
Lemma 2.7. Let $A^{\prime} \in M_{\Phi}$, and let $\kappa\left(A^{\prime}\right)$ be an infinite subfilament associated with $A^{\prime}$, such that $A^{\prime}$ is the only matrix on $\kappa\left(A^{\prime}\right)$ that may also be on a finite filament. Consider the map

$$
p: M_{\Phi} \longrightarrow M_{\Phi}
$$

such that

$$
p(A)= \begin{cases}A & \text { if } A \text { is on a finite filament, } \\ \Phi & \text { if } A \text { is on some } \kappa\left(A^{\prime}\right) \text { for some } A^{\prime} \in M_{\Phi}, \text { and } A \neq A^{\prime} .\end{cases}
$$

Let $P_{\Phi}=\operatorname{Im}(p)$, the image of $p$. Then the space $P_{\Phi}$ has the quotient topology induced by $p$.

Proof. It is clear $p$ is a surjection. Furthermore, since $M_{\Phi}$ has the discrete topology, then if $U$ is open in $P_{\Phi}$, it must be that $U$ is open in $M_{\Phi}$.

This new topological space consists of exactly those finite sequences of matrices that will allow $\Phi$ to escape the condition of partition regularity. Therefore, the sizes of these spaces offer a better characterization of the degree of partition regularity of a given matrix. Now consider the following corollary to Theorem 2.1.

Corollary 2.8. Let $\mathscr{D}_{\Phi}=\left\{A \in P_{\Phi}: \operatorname{apr}(A)=1\right\}$. Then $\left(\mathscr{D}_{\Phi}, \delta\right)$ is a metric space, and $\left(\mathscr{D}_{\Phi}, \mathscr{T}\right)$ is a discrete topological space.

Proof. By Theorem 2.1 we know that

$$
\delta:[\Phi] \mathrm{X}[\Phi] \longrightarrow \mathbb{R}
$$

is a metric on $[\Phi]$. Since $\mathscr{D}_{\Phi}$ is a subset of $[\Phi]$, we know that

$$
\delta: \mathscr{D}_{\Phi} \mathrm{X} \mathscr{D}_{\Phi} \longrightarrow \mathbb{R}
$$

is a metric on $\mathscr{D}_{\Phi}$. Thus, $\left(\mathscr{D}_{\Phi}, \delta\right)$ is a metric space. Since every subset of $\mathscr{D}_{\Phi}$ is contained in $M_{\Phi}$, then we know for every $A_{0}$ in $\mathscr{D}_{\Phi}$, there exists an open ball

$$
\mathscr{B}_{A_{0}, 1 / 2}=\left\{A_{0}\right\}
$$

Therefore, every singleton in $\mathscr{D}_{\Phi}$ is open, and we conclude that $\left(\mathscr{D}_{\Phi}, \mathscr{T}\right)$ is a discrete topological space.

Lemma 2.9. If $P_{\Phi}$ is compact, then it is finite. Similarly, if $\mathscr{D}_{\Phi}$ is compact, then it is finite.

Proof. We will demonstrate the result for $P_{\Phi}$. The proof works analogously for $\mathscr{D}_{\Phi}$. Let $P_{\Phi}$ be compact. Since the collection $\mathscr{F}_{F}$ consisting of all singletons in $P_{\Phi}$ forms an open cover of $P_{\Phi}$, there exists a finite subcover $\mathscr{F}^{\prime}$ contained in $\mathscr{F}^{\prime}$. Assume that $\mathscr{F}^{\prime}$ is a proper subcollection of $\mathscr{F}$. Then the set $\mathscr{Q}=\left\{A \in P_{\Phi}:\{A\} \in \mathscr{F}^{\prime}\right\}$ has power set $\mathscr{P}(2)$ equal to the set of all sets that may be formed by taking the union of elements of $\mathscr{F}^{\prime}$. Similarly, $\mathscr{R}=\left\{A \in P_{\Phi}:\{A\} \in \mathscr{F}\right\}$ has power set $\mathscr{P}(\mathscr{R})$ 
equal to the set of all sets that may be formed by taking the union of elements of $\mathscr{F}$. Since $\mathscr{F}^{\prime}$ is a proper subcollection of $\mathscr{F}$, it must be that $\mathscr{P}(2)$ is a proper subcollection of $\mathscr{P}(\mathscr{R})$. Thus, we can choose $U \in \mathscr{P}(\mathscr{R}) \backslash \mathscr{P}(2)$ that is not equal to the empty set. Then

$$
u=\bigcup_{B \in \mathscr{U}}\{B\},
$$

and so any subset $\{B\}$ is not in $\mathscr{P}(2)$. Thus, the matrix $B$ cannot be in any open set contained in $\mathscr{F}^{\prime}$, a contradiction. Therefore, $\mathscr{F}^{\prime}=\mathscr{F}$, and consequently, $\mathscr{F}$ is a finite open cover of $P_{\Phi}$. Since $\mathscr{F}$ is the collection of all singletons in $P_{\Phi}$, we conclude that $P_{\Phi}$ must be finite.

Notice that for any $\Phi$ in $\mathbb{M}_{m, n}(\mathbb{C})$, the topological spaces $M_{\Phi}, P_{\Phi}$, and $\mathscr{D}_{\Phi}$ are all Hausdorff. We can think of $\mathscr{D}_{\Phi}$ as the boundary set of $P_{\Phi}$, since no matrix in $P_{\Phi}$ can be "closer" to leaving $P_{\Phi}$ than those matrices with antiprogress 1 . This relationship between $P_{\Phi}$ and $\mathscr{D}_{\Phi}$ grants us the following theorem.

Theorem 2.10. For any partition regular matrix $\Phi$, the quotient space $P_{\Phi}$ is compact if and only if $\mathscr{D}_{\Phi}$ is finite.

Proof. Necessity of the statement follows from Lemma 2.9. To demonstrate sufficiency, recall that $P_{\Phi}$ consists of only finite filaments. Every $A$ in $\mathscr{D}_{\Phi}$ is the last matrix of a finite filament. For such an $A$, pick $P$ and $Q$ such that $A=P \Phi Q$, and represent $P$ and $Q$ with the finite sequence of elementary operations $\langle x\rangle_{i=1}^{l}$. Then we can think of the finite filament ending in $A$ as the finite, ordered set of matrices

$$
\left(\Phi,\langle x\rangle_{i=1}^{1}(\Phi),\langle x\rangle_{i=1}^{2}(\Phi), \ldots,\langle x\rangle_{i=1}^{l-1}(\Phi), A\right),
$$

where $\langle x\rangle_{i=1}^{n}(\Phi)$ represents the matrix produced by applying the first $n$ operations of $\langle x\rangle_{i=1}^{l}$ to $\Phi$. If we let

$$
\langle x\rangle_{A}(\Phi)=\left(\Phi,\langle x\rangle_{i=1}^{1}(\Phi),\langle x\rangle_{i=1}^{2}(\Phi), \ldots,\langle x\rangle_{i=1}^{l-1}(\Phi), A\right),
$$

then

$$
P_{\Phi}=\bigcup_{A \in \mathscr{D}_{\Phi}}\langle x\rangle_{A}(\Phi) .
$$

It follows that $P_{\Phi}$ contains a finite number of matrices if and only if $\mathscr{D}_{\Phi}$ is finite. Now let $\mathbb{X}$ denote the set of all open sets in $P_{\Phi}$. Since every singleton is open in $P_{\Phi}$, we know that $\mathbb{X}$ is finite if and only if $\mathscr{D}_{\Phi}$ is finite.

Assume that $\mathscr{D}_{\Phi}$ is finite. Let $\mathscr{F}$ be an open cover of $P_{\Phi}$ (without duplicates), and assume that $\mathscr{F}$ is not finite. Then $|\mathbb{X}|<|\mathscr{F}|$. Thus, $\mathscr{F}$ must contain more open sets of $P_{\Phi}$ than are in the set $\mathbb{X}$, a contradiction. We conclude that $\mathscr{F}_{F}$ must be finite, and since any open cover $\mathscr{F}$ is a subcover of itself, then it must be that every open cover of $P_{\Phi}$ contains a finite subcover. Thus, $P_{\Phi}$ is compact. 
Corollary 2.11. $\mathscr{D}_{\Phi}$ is compact if and only if $\mathscr{D}_{\Phi}$ is finite.

Proof. Necessity of the statement again follows from Lemma 2.9. So assume that $\mathscr{D}_{\Phi}$ is finite. Then, $P_{\Phi}$ is compact. Since $P_{\Phi} \backslash \mathscr{D}_{\Phi}$ is open in $P_{\Phi}$, then $\mathscr{D}_{\Phi}$ is closed. Therefore, $\mathscr{D}_{\Phi}$ is a closed subset of a compact space, and we conclude that $\mathscr{D}_{\Phi}$ is compact.

Theorem 2.12. Let $\Phi$ be a partition regular matrix, and let the set $\mathscr{D}_{\Phi}$ be infinite. Then $P_{\Phi}$ is the union of an infinite number of disjoint, compact subspaces.

Proof. Let $\mathscr{G}=\left\{G_{i}: i \in \mathbb{N}\right\}$ be a partition of $\mathscr{D}_{\Phi}$ into nonempty, disjoint and finite subsets. Since each subset is finite and $\mathscr{D}_{\Phi}$ contains an infinite number of elements, there must exist an infinite number of subsets in $\mathscr{G}$. Now let $B_{1} \subset P_{\Phi}$ be the set of all matrices on a filament that terminates with a matrix contained in $G_{1}$. Then for $i>1$, let $B_{i} \subset P_{\Phi}$ be the set of all matrices on a filament that terminates with a matrix contained in $G_{i}$, but are not contained in $\bigcup_{j=1}^{i-1} B_{j}$.

If a filament ends in a matrix $A \in G_{i}$, then there exists a pair of invertible matrices $P$ and $Q$, such that $A=P \Phi Q$, that can be represented by a finite sequence of elementary operations $\langle x\rangle_{i=1}^{l}$. Thus, the filament terminating with $A$ may be written as the finite, ordered set of matrices

$$
\langle x\rangle_{A}(\Phi)=\left(\Phi,\langle x\rangle_{i=1}^{1}(\Phi),\langle x\rangle_{i=1}^{2}(\Phi), \ldots,\langle x\rangle_{i=1}^{l-1}(\Phi), A\right),
$$

where $\langle x\rangle_{i=1}^{n}(\Phi)$ represents the matrix produced by applying the first $n$ operations of $\langle x\rangle_{i=1}^{l}$ to $\Phi$. Then, for all $i \in\{1,2,3, \ldots\}$, the set

$$
\bigcup_{A \in G_{i}}\langle x\rangle_{A}(\Phi)
$$

is the union of a finite number of finite sets, and therefore is also finite. Consequently, each $B_{i}$ is finite for all $i$ since,

$$
B_{i} \subseteq \bigcup_{A \in G_{i}}\langle x\rangle_{A}(\Phi)
$$

Now let $\mathbb{X}$ be the set of all open sets in $B_{i}$. Since $B_{i}$ is finite and has the discrete topology, $\mathbb{X}=\mathscr{P}\left(B_{i}\right)$, and is also finite. Assume that $\mathscr{F}$ is an open cover of $B_{i}$ that contains an infinite number of open sets. Since $\mathscr{F}$ is a collection of open sets of $B_{i}$, it must be that $\mathscr{F} \subseteq \mathbb{X}$. Thus, $|\mathscr{F}| \leq|\mathbb{X}|$, which contradicts the finite size of $\mathbb{X}$. So $\mathscr{F}$ must be finite, and every open cover of $B_{i}$ is finite. Thus, every open cover of $B_{i}$ contains a finite subcover, namely itself. Therefore, $B_{i}$ is a compact subspace of $P_{\Phi}$. Since $\left\{B_{i}: i \in \mathbb{N}\right\}$ is an infinite set of disjoint subspaces of $P_{\Phi}$ and $P_{\Phi}=\bigcup_{i \in \mathbb{N}} B_{i}$, it follows that $P_{\Phi}$ is the union of an infinite number of disjoint, compact subspaces. 


\section{Partition regular topological surfaces and graphs}

In this section, we will create a topological space that has geometry describing the degree of partition regularity for an associated topological surface, and then we will show how these spaces may also be created for an arbitrary, finite graph.

Every topological surface $\mathscr{Y}$ has a triangulation, and $\mathscr{Y}$ is compact if and only if it has a triangulation consisting of a finite number of triangles. So let $\mathscr{S}$ be a topological surface, and let $T(\mathscr{S})$ be a triangulation of $\mathscr{S}$. Then, the set of all vertices and edges in $T(\mathscr{S})$ form a connected graph $G$. Let $\left\{\Gamma_{i}: i \in \mathbb{N}\right\}$ be the collection of all orientations of $G$.

Proposition 3.1. The collection $\left\{\Gamma_{i}: i \in \mathbb{N}\right\}$ is finite if and only if the surface $\mathscr{Y}$ is compact.

Proof. For necessity, let $\left\{\Gamma_{i}: i \in \mathbb{N}\right\}$ be finite and assume $\mathscr{S}$ is not compact. Then $T(\mathscr{Y})$ does not consist of a finite number of triangles. Thus, there exists an infinite number of edges in $G$, each of which may be assigned one of two directions. Let $\Gamma_{0}$ be in $\left\{\Gamma_{i}: i \in \mathbb{N}\right\}$ with $\mathscr{K}$ as the edge index set. Now, let $\Gamma_{n}$ be the orientation of $G$ obtained by reversing only the direction of edge $n$ in $\Gamma_{0}$. Then the set $\left\{\Gamma_{n}: i \in \mathscr{K}\right\}$ is infinite. However,

$$
\left\{\Gamma_{n}: i \in \mathscr{K}\right\} \subset\left\{\Gamma_{i}: i \in \mathbb{N}\right\},
$$

which gives a contradiction.

For sufficiency, notice that if $\mathscr{Y}$ is compact, then $T(\mathscr{Y})$ contains a finite number of triangles. Therefore, $E$, the set of all edges in $G$, is a finite set. Since each edge may have one of two directions, then $\left|\left\{\Gamma_{i}: i \in \mathbb{N}\right\}\right|=2^{|E|}$.

Let $G$ be a finite graph and $\Gamma_{i}$ an orientation of $G$. We know that $D_{\Gamma_{i}}$, the oriented incidence matrix of $\Gamma_{i}$, is partition regular if and only if $\Gamma_{i}$ is strongly connected [Hogben and McLeod 2010, Theorem 2.4]. Here we consider the subcollection $\mathscr{C}$ of $\left\{\Gamma_{i}: i \in \mathbb{N}\right\}$ consisting of all strongly connected orientations of $G$.

Theorem 3.2. The collection $\mathscr{C}$, of all strongly connected orientations of $G$, is a nonempty and proper subcollection of $\left\{\Gamma_{i}: i \in \mathbb{N}\right\}$, for any triangulation of any topological surface $\mathscr{Y}$.

Proof. Let $T(\mathscr{Y})$ be a triangulation of some topological surface $\mathscr{Y}$. Then the graph $G$ consisting of all vertices and edges in $T(\mathscr{Y})$ is a connected graph. It is well known that a graph $G$ has a strongly connected orientation if and only if the edge connectivity of $G$ is greater than or equal to 2 . Therefore, $G$ associated with $T(\mathscr{Y})$ will have a strongly connected orientation if and only if it does not have edge connectivity equal to 1 . We know that an edge $\{i, j\}$ of $G$ must be an edge of at least one triangle. Now let

$$
w=\left\{v_{1},\{1,2\}, v_{2}, \ldots, v_{i},\{i, j\}, v_{j}, \ldots,\{n-1, n\}, v_{n}\right\}
$$


be a walk on $G$ that uses edge $\{i, j\}$. If we remove edge $\{i, j\}$, then we can define the walk

$$
w^{\prime}=\left\{v_{1},\{1,2\}, v_{2}, \ldots, v_{i},\{i, k\}, v_{k},\{k, j\}, v_{j}, \ldots,\{n-1, n\}, v_{n}\right\},
$$

where $v_{k}$ is the third vertex in some triangle containing edge $\{i, j\}$. Thus, $G \backslash\{\{i, j\}\}$ is still connected, and consequently, $G$ cannot have edge connectivity equal to 1 . Therefore, there exists a strongly connected orientation of $G$, and $\mathscr{C}$ is nonempty.

To see that $\mathscr{b} \neq\left\{\Gamma_{i}: i \in \mathbb{N}\right\}$, notice that for any vertex $v_{i}$ of $G$, there exists an orientation $\Gamma_{i}$ in $\left\{\Gamma_{i}: i \in \mathbb{N}\right\}$ such that any edge connected to $v_{i}$ has $v_{i}$ as its head. Thus, $\Gamma_{i}$ cannot be strongly connected, and $\mathscr{C}$ must be a proper subcollection of $\left\{\Gamma_{i}: i \in \mathbb{N}\right\}$.

Therefore, if $\mathscr{Y}$ is compact, then for each $\Gamma_{i}$ in $\mathscr{C}, D_{\Gamma_{i}}$ is partition regular. Furthermore, for each $D_{\Gamma_{i}}$ we can create the associated quotient space $P_{D_{\Gamma_{i}}}=P_{\Gamma_{i}}$.

Definition 3.3. Given a compact topological surface $\mathscr{Y}$, let $\mathfrak{T}$ be the set of all triangulations of $\mathscr{Y}$. For $t \in \mathfrak{T}$, let $\mathscr{C}_{t}$ be the set of all strongly connected orientations of the graph associated with $t$. Then $\mathscr{Y}$ is a partition regular surface if the product space

$$
\prod_{t \in \mathfrak{T}}\left(\prod_{\Gamma_{i} \in \mathscr{C}_{t}} P_{\Gamma_{i}}\right)
$$

is nonempty.

Theorem 3.4. Let $\mathscr{Y}$ be a compact topological surface. Then $\mathscr{Y}$ is partition regular. Proof. Recall that the set $\mathscr{C}_{t}$ is nonempty for any triangulation $t$, of any surface $\mathscr{T}$. Thus, there is at least one quotient space for each distinct triangulation of $\mathscr{Y}$ in the product topology associated with $\mathscr{S}$. Consequently, the product topology associated with $\mathscr{Y}$ is not an empty space, and thus $\mathscr{Y}$ is partition regular.

Definition 3.5. For any finite graph $G$, let $\mathscr{C}$ be the set of all strongly connected orientations of $G$. Then $G$ is a partition regular graph if the product space

$$
\prod_{\Gamma_{i} \in \mathscr{C}_{t}} P_{\Gamma_{i}}
$$

is nonempty.

In contrast to Theorem 3.4, the following theorem shows that not all finite graphs are partition regular.

Theorem 3.6. Let $G$ be a finite tree. Then $G$ is not a partition regular graph.

Proof. Since no orientation of a tree graph is strongly connected, then every quotient space in the product topology associated with a tree graph is empty. Consequently, any such product topology is empty, and no tree graph is a partition regular graph. 


\section{Conclusion}

As is captured in the contrasting scenarios presented in Theorems 3.4 and 3.6, partition regularity may be thought of as a property with varying degree that is dependent on the object being studied. For instance, we began with a compact topological surface $\mathscr{Y}$, traced the notion of order in the context of topological surfaces, through the graph theoretical context, and finally the matrix theoretical context. Consequently, we were able to construct topological spaces characterizing the degree of order for the surface $\mathscr{Y}$. We have also seen that every compact topological surface is a partition regular surface, and thus exhibits, as should be expected, some level of order. We may now explore the concept of partition regularity for compact topological surfaces and finite graphs. Moreover, we now possess structures that allow us to no longer think of an object as simply being partition regular, but instead, as having some degree of partition regularity. Subsequently, we may begin to relate matrices, graphs, and topological surfaces based on their relative degrees of partition regularity.

\section{Acknowledgements}

The author thanks the 2010 NSF REU Program at Mount Holyoke College, and Professor Jillian McLeod of Mount Holyoke College for her critical review of the material in this paper.

The author also thanks the faculty and staff of the Oberlin College Department of Mathematics.

\section{References}

[Hindman 2007] N. Hindman, "Partition regularity of matrices", pp. 265-298 in Combinatorial number theory (Carrollton, GA, 2005), edited by B. Landman et al., de Gruyter, Berlin, 2007. Also available as article \#A18 in Integers Elec. J. Combin. Number Theory 7:2 (2007), accessible from http://www.integers-ejcnt.org/vol7-2.html. MR 2008g:05216 Zbl 1125.05105

[Hogben and McLeod 2010] L. Hogben and J. McLeod, "A linear algebraic view of partition regular matrices”, Lin. Alg. Appl. 433 (2010), 1809-1820. Zbl 05811008

[Rado 1943] R. Rado, "Note on combinatorial analysis", Proc. London Math. Soc. 48 (1943), 122 160. MR 5,87a Zbl 0028.33801

Received: 2010-08-02 Revised: 2010-12-21 Accepted: 2010-12-22

Isolus@oberlin.edu

Department of Mathematics, Oberlin College, OCMR 2293, 135 W Lorain Street, Oberlin, OH 44074, United States 


\section{involve}

pjm.math.berkeley.edu/involve

\section{EDITORS}

MANAGING EDITOR

Kenneth S. Berenhaut, Wake Forest University, USA, berenhks@wfu.edu

\section{BOARD OF EDITORS}

John V. Baxley Wake Forest University, NC, USA baxley@wfu.edu

Arthur T. Benjamin Harvey Mudd College, USA benjamin@hmc.edu

Martin Bohner Missouri U of Science and Technology, USA bohner@mst.edu

Nigel Boston University of Wisconsin, USA boston@math.wisc.edu

\section{Amarjit S. Budhiraja U of North Carolina, Chapel Hill, USA} budhiraj@email.unc.edu

Pietro Cerone Victoria University, Australia pietro.cerone@vu.edu.au

Scott Chapman Sam Houston State University, USA scott.chapman@shsu.edu

Jem N. Corcoran University of Colorado, USA corcoran@colorado.edu

Michael Dorff Brigham Young University, USA mdorff@math.byu.edu

Sever S. Dragomir Victoria University, Australia sever@matilda.vu.edu.au

Behrouz Emamizadeh The Petroleum Institute, UAE bemamizadeh@pi.ac.ae

Errin W. Fulp Wake Forest University, USA fulp@wfu.edu

Andrew Granville Université Montréal, Canada andrew@dms.umontreal.ca

Jerrold Griggs University of South Carolina, USA griggs@math.sc.edu

Ron Gould Emory University, USA rg@mathcs.emory.edu

Sat Gupta U of North Carolina, Greensboro, USA sngupta@uncg.edu

Jim Haglund University of Pennsylvania, USA jhaglund@math.upenn.edu

Johnny Henderson

Natalia Hritonenko

Charles R. Johnson

Karen Kafadar Baylor University, USA johnny_henderson@baylor.edu Prairie View A\&M University, USA nahritonenko@pvamu.edu

College of William and Mary, USA crjohnso@math.wm.edu

University of Colorado, USA karen.kafadar@cudenver.edu

K. B. Kulasekera Clemson University, USA

kk@ces.clemson.edu

Gerry Ladas University of Rhode Island, USA gladas@math.uri.edu

David Larson Texas A\&M University, USA larson@math.tamu.edu

Suzanne Lenhart University of Tennessee, USA lenhart@math.utk.edu
Chi-Kwong Li

Robert B. Lund

Gaven J. Martin

Mary Meyer

Emil Minchev

Frank Morgan

Mohammad Sal Moslehian

Zuhair Nashed

Ken Ono

Joseph O'Rourke

Yuval Peres

Y.-F. S. Pétermann

Robert J. Plemmons

Carl B. Pomerance

Bjorn Poonen

James Propp

Józeph H. Przytycki

Richard Rebarber

Robert W. Robinson

Filip Saidak

Andrew J. Sterge

Ann Trenk

Ravi Vakil

Ram U. Verma

John C. Wierman
College of William and Mary, USA ckli@math.wm.edu

Clemson University, USA lund@clemson.edu

Massey University, New Zealand g.j.martin@massey.ac.nz

Colorado State University, USA meyer@stat.colostate.edu

Ruse, Bulgaria eminchev@hotmail.com

Williams College, USA frank.morgan@williams.edu

Ferdowsi University of Mashhad, Iran moslehian@ferdowsi.um.ac.ir

University of Central Florida, USA znashed@mail.ucf.edu

University of Wisconsin, USA ono@math.wisc.edu

Smith College, USA orourke@cs.smith.edu

Microsoft Research, USA peres@microsoft.com

Université de Genève, Switzerland petermann@math.unige.ch

Wake Forest University, USA plemmons@wfu.edu

Dartmouth College, USA carl.pomerance@dartmouth.edu UC Berkeley, USA poonen@math.berkeley.edu U Mass Lowell, USA jpropp@cs.uml.edu

George Washington University, USA przytyck@gwu.edu

University of Nebraska, USA rrebarbe@math.unl.edu

University of Georgia, USA rwr@cs.uga.edu

U of North Carolina, Greensboro, USA f_saidak@uncg.edu

Honorary Editor andy@ajsterge.com

Wellesley College, USA atrenk@wellesley.edu

\section{Stanford University, USA} vakil@math.stanford.edu

University of Toledo, USA verma99@msn.com

Johns Hopkins University, USA wierman@jhu.edu

\section{PRODUCTION}

See inside back cover or http://pjm.math.berkeley.edu/involve for submission instructions.

The subscription price for 2010 is US \$100/year for the electronic version, and \$120/year (+\$20 shipping outside the US) for print and electronic. Subscriptions, requests for back issues from the last three years and changes of subscribers address should be sent to Mathematical Sciences Publishers, Department of Mathematics, University of California, Berkeley, CA 94704-3840, USA.

Involve (ISSN 1944-4184 electronic, 1944-4176 printed) at Mathematical Sciences Publishers, Department of Mathematics, University of California, Berkeley, CA $94720-3840$ is published continuously online. Periodical rate postage paid at Berkeley, CA 94704, and additional mailing offices.

Involve peer review and production are managed by EditFLOW ${ }^{\mathrm{TM}}$ from Mathematical Sciences Publishers.

\section{mathematical sciences publishers \\ http://www.mathscipub.org \\ A NON-PROFIT CORPORATION \\ Typeset in LATEX $_{\mathrm{E}}$ \\ Copyright $@ 2010$ by Mathematical Sciences Publishers}




\section{involve $2010 \quad$ vol. $3 \quad$ no. 4}

Identification of localized structure in a nonlinear damped harmonic oscillator using

Hamilton's principle

THOMAS VOGEL AND RYAN ROGERS

Chaos and equicontinuity

SCOTT LARSON

Minimum rank, maximum nullity and zero forcing number for selected graph families

Edgard Almodovar, Laura Deloss, Leslie Hogben, Kirsten Hogenson,

Kaitlyn Murphy, Travis Peters and Camila A. Ramírez

A numerical investigation on the asymptotic behavior of discrete Volterra equations with

two delays

ImMacolata Garzilli, Eleonora Messina AND ANTONia Vecchio

Visual representation of the Riemann and Ahlfors maps via the Kerzman-Stein equation Michael Bolt, Sarah SNOEYINK AND Ethan VAN ANDEl

A topological generalization of partition regularity

LIAM SOLUS

Energy-minimizing unit vector fields

Yan Digilov, William Eggert, Robert Hardt, James Hart, Michael

Jauch, Rob Lewis, Conor Loftis, Aneesh Mehta, Hector Perez,

LeObardo Rosales, ANANd ShaH ANd Michael Wolf

Some conjectures on the maximal height of divisors of $x^{n}-1$

NATHAN C. RYAN, BRYAN C. WARD AND RYAN WARD

Computing corresponding values of the Neumann and Dirichlet boundary values for incompressible Stokes flow

John Loustau AND Bolanle Bob-EgBE 